\title{
Retrieval Comparison of EndNote to Search MEDLINE (Ovid and PubMed) versus Searching Them Directly
}

\author{
Carole Gall \\ Frances A. Brahmi
}

\begin{abstract}
Using EndNote version 7.0, the authors tested the search capabilities of the EndNote search engine for retrieving citations from MEDLINE for importation into EndNote, a citation management software package. Ovid MEDLINE and PubMed were selected for the comparison. Several searches were performed on Ovid MEDLINE and PubMed using EndNote as the search engine, and the same searches were run on both Ovid and PubMed directly. Findings indicate that it is preferable to search MEDLINE directly rather than using EndNote. The publishers of EndNote do warn its users about the limitations of their product as a search engine when searching external databases. In this article, the limitations of EndNote as a search engine for searching MEDLINE were explored as related to MeSH, non-MeSH, citation verification, and author searching. [Article copies available for a fee from The Haworth Document Delivery Service: 1-800-HAWORTH. E-mail address: $<$ docdelivery@haworthpress.com> Website: <http://www.HaworthPress.com> (C) 2004 by The Haworth Press, Inc. All rights reserved.]
\end{abstract}

Carole Gall, MLS (cfgall@iupui.edu) is Development Officer \& Manager Print Journal Collection, and Frances A. Brahmi, MA, MLS, AHIP (fbrahmi@iupui.edu) is Curriculum \& Education Director, both at Ruth Lilly Medical Library, Indiana University School of Medicine, 975 West Walnut Street, IB Rm 322, Indianapolis, IN 46202-5121. Ms. Brahmi is also Director of Lifelong Learning, Indiana University School of Medicine.

Medical Reference Services Quarterly, Vol. 23(3), Fall 2004

http://www.haworthpress.com/web/MRSQ

(C) 2004 by The Haworth Press, Inc. All rights reserved. Digital Object Identifier: 10.1300/J115v23n03_03 
KEYWORDS. EndNote, MEDLINE, Ovid, PubMed, citation management software

\section{INTRODUCTION}

The purpose of this article is to demonstrate the differences of output from searching MEDLINE direct and searching MEDLINE using EndNote. The authors were interested in the pitfalls of using EndNote as the search intermediary to retrieve citations from MEDLINE versus searching MEDLINE direct, using either PubMed or Ovid. Because searching via EndNote is convenient in terms of one-stop shopping, so to speak, it might be very tempting to the untrained searcher. In addition, searching from EndNote is fairly straightforward and simple. Expert searchers avail themselves of sophisticated tools in Ovid and PubMed, but can that kind of searching be done via EndNote? Is it ever appropriate to use the EndNote search features for MEDLINE? How do the search results differ?

\section{METHODOLOGY}

Four searches were conducted using PubMed via EndNote (EN), PubMed direct, Ovid via EndNote, and Ovid direct. The four searches were as follows: Medical Subject Heading (MeSH) combined with free-text; MeSH combined with Author; Non-MeSH; and known citation verification. Ovid and PubMed were selected because they are the two most commonly used versions of MEDLINE in academic and hospital settings. PubMed was searched the simplest way via the National Library of Medicine and Ovid MEDLINE from 1966 through Week 2 of November 2003. The study was limited to the evaluation of output, given the searching capabilities of each system. EndNote was not addressed as a citation manager, but rather as a way to search external databases in order to build a citation library. The EndNote manual cautions its users that the EndNote search interface is meant for simple searches, and "not to replace the advanced search options" offered by MEDLINE information providers. Actually, the EndNote manual provides an extensive, up-to-date table of Output Formats and Corresponding Import Options to assist its users with downloading instructions for 29 databases. 


\section{RESULTS}

Combined Author and MeSH Term searching is a fairly common request. In putting the search together, four different ways were tried, as seen in Table 1. L. H. Einhorn is a local researcher who is recognized internationally for his work on testicular cancer. He publishes both as L. H. and L. Einhorn. As can be seen from Table 1, under EndNote PubMed, using Einhorn (qualified to author) and ANDing it with testicular (qualified to Keywords), resulted in no retrieval. This is because testicular is not a MeSH term. However, if testicular is qualified to Any Field, EndNote PubMed retrieves 161 articles. If Einhorn, LH (qualified to author) is ANDed to testicular (qualified to Any field), the retrieval is 158. Looking across the rows, it is easy to discern how confusing this might be for a user. Similarly, the same is true for EndNote Ovid searching.

The same author and MeSH term were searched in Ovid MEDLINE, and the three different search strategies appear in Table 2. The first three sets in Table 2 show the author and MeSH term ANDed together to complete one search strategy. Sets four and five demonstrate additional strategies, each complete in themselves. Set four uses a " $\$$ " as a wildcard for the initial of the author, and uses ".mp." to map the term to a title, abstract, Chemical Abstracts Service Registry number or Enzyme Commission number, and MeSH heading.

The entry terms "lyme disease AND children" were searched as follows: "lyme disease" was searched both as a MeSH term and as text word, and "children" was searched as a text word and as the MeSH term "child." Table 3 shows the varied results again using EndNote PubMed, EndNote Ovid MEDLINE, PubMed, and Ovid MEDLINE.

The strategy for searching PubMed directly is included in Table 3. The strategy for searching Ovid MEDLINE directly is in Table 4.

\section{Non-Medical Subject Heading (MeSH)}

The non-MeSH term, GATA-3, a zinc finger protein that is preferentially expressed in T cells in adult animals, was selected because it is alpha-numeric and includes a hyphen, traditionally a challenging format for most database systems. This topic was searched as GATA-3, GATA 3 , and GATA3.

As shown in Table 5, GATA-3 retrieved identical results (245 hits) when searching PubMed directly or via EndNote (qualified to Any 
TABLE 1. Combined Author and MeSH Term

\begin{tabular}{|c|c|c|c|c|}
\hline $\begin{array}{l}\text { Combined Author } \\
\text { and Subject Search } \\
\text { (MeSH) 12/10/03 }\end{array}$ & $\begin{array}{l}\text { Einhorn } \\
\text { AND } \\
\text { testicular }\end{array}$ & $\begin{array}{l}\text { Lh Einhorn } \\
\text { AND testicular- } \\
\text { neoplasms }\end{array}$ & $\begin{array}{l}\text { Einhorn AND } \\
\text { testicular- } \\
\text { neoplasms }\end{array}$ & $\begin{array}{l}\text { Einhorn Ih* } \\
\text { AND testicular }\end{array}$ \\
\hline EndNote PubMed & 0 & 149 & 151 & 0 \\
\hline Author \& Keywords & 161 & 149 & 151 & 158 \\
\hline Author \& Any Field & 151 & 136 & 151 & 136 \\
\hline EndNote MEDLINE Ovid & 161 & 136 & 151 & 143 \\
\hline Author \& Keywords & 181 & 3 & 168 & 142 \\
\hline Author \& Any Field & 136 & 136 & 151 & (no comma) \\
\hline $\begin{array}{c}\text { PubMed } \\
\text { (no comma) }\end{array}$ \\
\hline $\begin{array}{l}\text { Ovid MEDLINE- } \\
\text { See below for strategies }\end{array}$ & 159 & & 79 \\
\hline
\end{tabular}

*The comma after Einhorn was either included or not depending on the requirements of the system tested.

TABLE 2. Ovid MEDLINE 1966 to November Week 22003

\begin{tabular}{|l|l|c|}
\hline$\#$ & \multicolumn{1}{|c|}{ Search History } & Results \\
\hline 1 & Testicular Neoplasms/ & 14871 \\
\hline 2 & Einhorn Ih.au. & 297 \\
\hline 3 & 1 and 2 & 136 \\
\hline 4 & Einhorn \$ (au) and testicular neoplasms.mp.** & 151 \\
\hline 5 & Einhorn lh $(\mathrm{au})$ and testicular.mp. ${ }^{* *}$ & 79 \\
\hline
\end{tabular}

${ }^{\star *} \mathrm{Mp}=$ map to title, abstract, CAS registry/ec number, $\mathrm{MeSH}$.

TABLE 3. Combined MeSH and Term and Free Text

\begin{tabular}{|c|c|}
\hline Combined Subject Search (MeSH) AND free-text & $\begin{array}{c}\text { Lyme Disease } \\
\text { AND Children }\end{array}$ \\
\hline EndNote PubMed & 863 \\
\hline Keyword \& Any Field & 901 \\
\hline Any Field \& Any Field & \\
\hline EndNote Ovid MEDLINE & 232 \\
\hline Keyword \& Any Field & 253 \\
\hline Any Field \& Any Field & 921 \\
\hline PubMed direct-(lyme disease AND children) & 781 \\
\hline Ovid MEDLINE direct-see Table 4 for strategy & \\
\hline
\end{tabular}


TABLE 4. Ovid MEDLINE Direct 1966 to November Week 22003

\begin{tabular}{|l|l|c|}
\hline$\#$ & Search History & Results \\
\hline 1 & Lyme Disease/ & 5662 \\
\hline 2 & Child/ (entry word: children) & 870850 \\
\hline 3 & 1 and 2 & 781 \\
\hline
\end{tabular}

TABLE 5. GATA-3, Non-MeSH Search Term with Two Variants

\begin{tabular}{|l|c|c|c|}
\hline Non-MeSH Search & GATA-3 & GATA 3 Variant & GATA3 Variant \\
\hline EndNote PubMed & & & \\
\hline Any Field & 245 & 2,591 & 58 \\
\hline Keywords & 0 & 0 & 0 \\
\hline EndNote Ovid MEDLINE & & & 58 \\
\hline Any Field & 0 & 0 & 0 \\
\hline Keywords & 0 & 0 & 58 \\
\hline PubMed direct & 245 & 1,007 & 59 \\
\hline Ovid MEDLINE direct & 297 & 315 & \\
\hline
\end{tabular}

Field). The same search in Ovid MEDLINE via EndNote retrieved zero, while searching Ovid MEDLINE directly yielded 297 hits.

GATA 3 (without the hyphen) yielded zero or mostly false drops, with the exception of the direct Ovid MEDLINE search, where the term was searched as "GATA 3.mp." and yielded 315 hits. Using GATA3 (no space) yielded 58 articles in PubMed via EndNote (qualified to Any Field) or directly, whereas Ovid MEDLINE via EndNote and Ovid MEDLINE directly yielded 58 and 59 articles, respectively.

\section{Citation Verification}

A common way of using a citation management system is to search for and save a known reference. The authors chose to include a knownreference search in the comparison, and used a reference that was five months old at the time of the search.

Houghton, J.; George, W.D.; Cuzick, J.; Duggan, C.; Fentiman, I.S.; Spittle, M. Radiotherapy and tamoxifen in women with completely excised ductal carcinoma in situ of the breast in the UK, Australia, and New Zealand: randomised controlled trial. Lancet. 362(9378):95-102, 2003 Jul 12. 
To do a known-reference search, the authors chose to use the title field, and typed in the first eight words of the title of the article. Searches in EndNote PubMed and PubMed direct both yielded six articles: the exact citation and five more recent citations, which were in response to the original article. EndNote Ovid MEDLINE and Ovid MEDLINE direct both yielded only the exact citation. Alternatively, using free-text searching (qualifying to title) and the journal name field in Ovid direct retrieved the article of interest, as shown in Table 6.

\section{DISCUSSION}

\section{Connecting to a Remote Database}

When connecting from EndNote to a remote database, the searcher must select the remote database from an impressive list of possibilities. Online, EndNote provides access to over 120 databases (excluding library catalogs). The databases are listed alphabetically from ABI Inform (OCLC FirstSearch) to Zoo Record Plus (Cambridge Scientific Abstracts). Not all MEDLINE databases are grouped together. Using the Find box, entering MEDLINE retrieves all MEDLINE and PreMEDLINE, but does not retrieve PubMed. This might lead the user to think that PubMed is not an option. The authors, both trained search analysts, found this to be confusing and thus anticipated that it would prove even more confusing to an end-user searcher. When teaching end users about EndNote, it is something that should be pointed out.

\section{Using the Search Box}

A searcher can define a search field in EndNote by clicking Add Field, but in comparison to PubMed and Ovid, the options for refined

TABLE 6. Ovid MEDLINE 1966 to November Week 22003

\begin{tabular}{|l|l|c|}
\hline$\#$ & \multicolumn{1}{|c|}{ Search History } & Results \\
\hline 1 & tamoxifen.ti. & 4498 \\
\hline 2 & lancet.jn. & 100763 \\
\hline 3 & ductal carcinoma.ti. & 955 \\
\hline 4 & 1 and 2 and 3 & 1 \\
\hline
\end{tabular}


searching are few. The search strategy can be saved by clicking Save Search, as can be done in PubMed and Ovid. EndNote lacks the limits that are easily accessible in Ovid and PubMed, for advanced search options.

When using the Delete Field in EndNote, the top search field disappears rather than the last search field, as one would expect. Additionally, there is no Undo command. With an experienced EndNote user, this may not be a problem.

Also, the Close and Search boxes on the Search screen should be reversed, for more intuitive use. The same is true with the boxes for Cancel and Connect on the "Choose a Connection" File screen.

When adjusting search strategy, running a search, and readjusting, etc., the authors had to check with some regularity to be sure the remote database was being searched, and if not, the search had to be started all over again. To determine which is being searched, the searcher must look for a checkmark in the Search Remote box on the Search screen.

\section{Retrieving the Search Results}

The download time of large search results is an issue in EndNote and a significant difference when contrasting EndNote with information providers' databases. In EndNote7, a search with 2,591 results took 24 3/4 minutes to download into the preview box of EndNote on a Thursday at 8:50 a.m. When the same search was repeated and downloaded at 3:26 p.m. on the same day, it took exactly the same amount of time to download the 2,591 results. In comparison, results of 100,000 plus references in PubMed and Ovid MEDLINE are available in less than ten seconds. These searches, as shown in Table 7, were done on a 10/100 Base-T Internet connection on a Dell OptiPlex GX110.

The number of search results displays immediately in the Confirm Remote Search box, at which time a searcher's choices are to limit the number of citations to download, or Cancel the search, which flips back to the Search box. At that time, the searcher can both refine and rerun the original search, though a restricted number of limits and enhancements are available in EndNote7. However, once the download begins, the searcher has the option to preview the citations by clicking the Show Preview box, or to Pause and double click a citation to see the full record. The download can continue by clicking Resume. 
TABLE 7. Download Time of Search Results in EndNote

\begin{tabular}{|c|c|}
\hline Number of Citations Downloaded & Minutes to Download \\
\hline 500 & $41 / 4$ \\
\hline 1,000 & $83 / 4$ \\
\hline 1,500 & $131 / 4$ \\
\hline 2,000 & $181 / 2$ \\
\hline 2,500 & $231 / 4$ \\
\hline 2,591 & $243 / 4$ \\
\hline
\end{tabular}

\section{CONCLUSION}

EndNote provides an impressive list of remote bibliographic databases to connect to, but the search mechanism is structured for basic searches. In comparison to Ovid MEDLINE and PubMed, a restricted number of limits and enhancements are available in EndNote7, although it includes an easy-to-use mechanism to save searches for later rerun, and a default search setting. It is an efficient way to download a known citation.

Until experienced, the arrangement of PubMed separate from the grouping of MEDLINE as a remote database option is a problem. In order to perfect a search strategy, the authors found that it takes experience to know whether one is searching the remote database or the internal EndNote library. In EndNote, searchers will want to structure MEDLINE searches to retrieve a small number of results; otherwise, they face a lengthy time for downloading results, which is an unfavorable comparison to direct searching in PubMed and Ovid MEDLINE.

The authors do not recommend using EndNote for searching MEDLINE. The convenience is far outweighed by the confusing variety of results obtained. Rather, it is recommended that searching be done using the MEDLINE direct method (either PubMed or Ovid) and that the resulting output be imported into EndNote.

Received: January 2, 2004

Revised: January 12, 2004

Accepted: January 14, 2004 